\title{
Heparinas de bajo peso molecular en pacientes con enfermedad renal crónica ¿Es seguro su uso?
}

\author{
JORGE VEGA S. ${ }^{1,2,3}$, GONZALO MARTÍNEZ R. ${ }^{1,2}$, HELMUTH GOECKE S. ${ }^{1,2}$
}

\section{Are low-molecular-weight heparins safe in patients with chronic kidney disease?}

Due to their efficacy and convenience, low-molecular-weight heparins (LMWH) are used as sustitutes of unfractionated heparin. Unfortunately, most of the evidence about safety and usefulness of LMWH have excluded patients with chronic kidney disease (CKD), in whom their elimination clearance is reduced, allowing an increased anticoagulant effect. Accordingly, there is a growing number of reports about major and fatal bleeding episodes in this group of patients using LMWH. At the present stage of knowledge, there is no definitive cut-off value of renal function to adjust the doses or avoid the administration of LMWH, making their effects unpredictable in patients with CKD. Hence, it is reasonable to avoid the use of these drugs in patients with CKD, while awaiting for more evidence that supports their safer use.

(Rev Med Chile 2010; 138: 487-495). chronic.

Key words: Heparin, low-molecular weight; Kidney diseases; Kidney failure,

L a heparina no fraccionada (HNF) ha sido por décadas la droga anticoagulante utilizada tanto en la profilaxis como en la terapia de la enfermedad tromboembólica, así como en la prevención de la coagulación de los circuitos extracorpóreos en procedimientos como la hemodiálisis.

En los últimos años las heparinas de bajo peso molecular (HBPM) han ido reemplazando a la HNF en muchas de sus indicaciones, gracias a una serie de ventajas (Tabla 1) ${ }^{1-3}$. Las HBPM han demostrado ser superiores a la HNF en síndromes coronarios agudos, donde se ha observado $17 \%$ de reducción en el riesgo de muerte e infarto no fatal ${ }^{2}$ al igual que en casos de tromboembolismo venoso agudo, donde se ha asociado con menor frecuencia a trombosis recurrente y muerte ${ }^{1}$.

Aparentemente, estas ventajas de las HBPM no se acompañan de mayor riesgo de hemorragias, con una incidencia de sangrados mayores de $1,1 \%$ para HNF y de $1,3 \%$ para enoxaparina ${ }^{4}$. Sin embargo, la mayoría de los estudios han excluido a sujetos con obesidad mórbida, ancianos, niños, embarazadas y a los pacientes con enfermedad renal crónica (ERC) en etapas 3 a 5 (Tabla 2), debido a la farmacocinética impredecible de las HBPM en estos grupos de pacientes ${ }^{5,6}$.

En los últimos años, en pacientes con insuficiencia renal se han descrito en forma creciente complicaciones hemorrágicas graves, incluso letales, con el uso de HBPM. Se han comunicado sangrados digestivos y hematomas de la pared abdominal, retroperitoneales, peri-renales, cerebrales, oculares, pericárdicos, del músculo ilio-psoas y epidurales ${ }^{7-26}$.

Dado el aumento progresivo de los pacientes con ERC y el empleo cada vez más extendido de las HBPM en ellos, creímos necesario revisar y comunicar la evidencia existente sobre el uso de HBPM en ERC, para advertir sobre sus riesgos y reducir la aparición de complicaciones hemorrágicas graves. La evidencia disponible del uso de las HBPM en 
insuficiencia renal aguda y en trasplante renal no fue incluida en esta revisión.

\section{Estructura química y mecanismos de acción de las heparinas}

La HNF consiste en una mezcla heterogénea de glicosaminoglicanos ramificados polianiónicos de distintos pesos moleculares, que varían entre 3.000 y 30.000 daltons (media de 15.000 daltons). Es extraída desde la mucosa intestinal porcina o de pulmones bovinos. Las HBPM son preparadas desde la HNF a través de depolimerización química o enzimática y tienen peso molecular entre

\section{Tabla 1. Ventajas del uso de heparinas de bajo peso molecular}

Facilidad de uso (se administra por vía subcutánea)

Efecto predecible (no requiere de monitoreo de laboratorio)

Administración en dosis fija según peso corporal

Posibilidad de uso ambulatorio (acorta o evita las hospitalizaciones)

Reduce los costos totales de la terapia anticoagulante

Vía media de eliminación prolongada (se usa 1 a 2 veces al día)

Menor riesgo de trombocitopenia asociada a heparina

Menor riesgo de osteoporosis

Mayor disponibilidad (menor unión a proteínas y a células endoteliales, macrófagos, osteoblastos y plaquetas)
3.000 y 9.000 daltons (media de 5.000 daltons) ${ }^{27}$.

La biodisponibilidad de la HNF es baja en dosis reducidas (30\% luego de un bolo). Debido a su estructura polianiónica y a las largas cadenas de polisacáridos, la HNF se une no sólo a la antitrombina sino que también a otras proteínas y membranas en forma inespecífica y saturable, en una proporción variable y difícil de predecir. Por ello un bolo inicial de HNF debe contener una dosis suficiente para saturar estas uniones a sitios inespecíficos, luego de lo cual hay una respuesta casi lineal entre dosis y respuesta anticoagulante. Las HBPM en cambio tienen una biodisponibilidad cercana a $100 \%$ cuando se administran por vía subcutánea ${ }^{3}$ al unirse en menor proporción a las células endoteliales y proteínas plasmáticas ${ }^{27}$.

La HNF es rápidamente metabolizada en el sistema retículo endotelial y menos de $10 \%$ es excretado inalterado en la orina ${ }^{28}$. Las HBPM no son aclaradas por el hígado y su clearence renal es más lento ${ }^{29}$. Mientras más pequeño es el peso molecular de la HBPM (como la enoxaparina), más lenta es su eliminación ${ }^{30}$.

La secuencia química clave para la unión de la HNF a la antitrombina es un pentasacárido compuesto de 3 glucosaminas sulfatadas y de dos ácidos urónicos. Estos pentasacáridos se unen y activan a la antitrombina, potenciando su inhibición del factor Xa y de la trombina ${ }^{27}$. Los pentasacáridos se encuentran en un tercio de la HNF y entre $5 \%$ y $15 \%$ de las $\mathrm{HBPM}^{29}$. Cualquier cadena de heparina que tenga la secuencia única de pentasacáridos puede unirse a la antitrombina, produciendo un cambio conformacional en esta última que permite inhibir la actividad del factor Xa. Sin

Tabla 2. Clasificación de la enfermedad renal crónica

\begin{tabular}{|c|c|c|}
\hline Etapa* & Descripción & Velocidad de filtración glomerular \\
\hline 1 & $\begin{array}{l}\text { Velocidad de filtración glomerular normal o elevada y evidencias } \\
\text { de "daño renal"** }\end{array}$ & $\geq 90 \mathrm{ml} / \mathrm{min} / 1,73 \mathrm{~m}^{2}$ \\
\hline 2 & $\begin{array}{l}\text { Reducción de la filtración glomerular leve y evidencias de "daño } \\
\text { renal". }\end{array}$ & $60-89 \mathrm{ml} / \mathrm{min} / 1,73 \mathrm{~m}^{2}$ \\
\hline 3 & $\begin{array}{l}\text { Reducción de la filtración glomerular moderada con o sin evi- } \\
\text { dencias de "daño renal" }\end{array}$ & $30-59 \mathrm{ml} / \mathrm{min} / 1,73 \mathrm{~m}^{2}$ \\
\hline 4 & Reducción severa de la velocidad de filtración glomerular & $15-29 \mathrm{ml} / \mathrm{min} / 1,73 \mathrm{~m}^{2}$ \\
\hline 5 & Falla renal & $<15 \mathrm{ml} / \mathrm{min} / 1,73 \mathrm{~m}^{2}$ (o diálisis) \\
\hline
\end{tabular}

* Alteraciones persistentes por al menos 3 meses.

**Definido como la presencia de daño patológico o marcadores de daño en exámenes de sangre, orina o imágenes. 
embargo, para inactivar a la trombina, la heparina debe formar un complejo terciario uniéndose tanto a la antitrombina como a la trombina. Este complejo sólo puede formarse por cadenas de heparinas que tengan al menos 18 unidades de sacáridos de longitud, incluyendo la secuencia de pentasacáridos. La mayoría de las cadenas de la HNF que contienen la secuencia de pentasacáridos son de largo suficiente, en cambio, menos de la mitad de las HBPM que contienen la secuencia de pentasacáridos son lo suficientemente largas para unirse simultáneamente a la antitrombina como a la trombina. Como resultado, la HNF tiene una actividad equivalente contra el factor Xa y la trombina (IIa), con una relación anti Xa/ anti IIa de 1:1. En contraste, las HBPM tienen una mayor actividad anti-Xa con una razón anti-Xa/ anti-IIa entre 4:1 a 2:127,29. En la enoxaparina esta razón es 4,1, en nadroparina y reviparina 3,5 , en dalteparina 2,4 y en tinzaparina 1,9. Como cada HBPM tiene diferentes pesos moleculares, densidad de cargas, características farmacocinéticas, clearance renal y actividad anti-Xa, ellas no son intercambiables y deben ser consideradas como drogas diferentes ${ }^{3,27,29-35}$.

\section{HBPM y enfermedad renal crónica en etapas 3 y 4}

Una de las principales limitaciones de todas las HBPM es que son mayoritariamente aclaradas por vía renal, por lo que su vida media se prolonga en pacientes con insuficiencia renal, aumentando la actividad anti-Xa $\mathrm{X}^{3,36,37}$. Aunque el uso de HBPM no está estrictamente contraindicado en pacientes con ERC, actualmente no hay datos que indiquen una efectividad y seguridad superior a la HNF, de modo que las ventajas que tienen las HBPM en sujetos con función renal normal no pueden extrapolarse a los sujetos con ERC $\mathrm{ER}^{3}$.

En los pacientes con nefropatías crónicas, las HBPM tienen un aclaramiento más lento y efectos anticoagulantes más prolongados, aumentando el riesgo de sangrado ${ }^{38}$. Existe una correlación inversa entre el clearance de creatinina y la actividad anti$\mathrm{X}$ a con el uso de enoxaparina y nadroparina. Con el uso de tinzaparina no se ha demostrado esta correlación ${ }^{38-42}$. Esta droga es estructuralmente parecida a la HNF y menos proclive a acumularse en pacientes con ERC que las HBPM más pequeñas y menos negativamente cargadas como la enoxaparina ${ }^{42}$.

Respecto a la enoxaparina, los pacientes con clearance de creatinina $<40 \mathrm{ml} / \mathrm{min}$ tienen una mayor actividad peak y valle anti-Xa que los sujetos con función renal normal, indicando acumulación de la droga ${ }^{43}$. En sujetos con clearance de creatinina $<30 \mathrm{ml} / \mathrm{min}$ la actividad anti-Xa aumenta un $65 \%{ }^{44}$. Cuando se usan al menos 3 dosis de enoxaparina de $1 \mathrm{mg} / \mathrm{kg}$ cada 12 horas en pacientes con clearance de creatinina $\leq 30 \mathrm{ml} / \mathrm{min}$, los niveles promedios de actividad anti-Xa varían entre 1,27 y $1,58 \mathrm{IU} / \mathrm{ml}$, en cambio en los sujetos con clearance de creatinina $>30 \mathrm{ml} / \mathrm{min}$, estos varían entre 0,91 y $1,06 \mathrm{IU} / \mathrm{ml}^{45}$. En un estudio, todos los pacientes recibieron una dosis inicial de enoxaparina de $1 \mathrm{mg} / \mathrm{kg}$, reduciéndose a $0,5 \mathrm{mg} / \mathrm{kg}$ cada 12 horas en los con clearance de creatinina $<$ $30 \mathrm{ml} / \mathrm{min}$ y $0,75 \mathrm{mg} / \mathrm{kg}$ en los con una depuración de creatinina entre 30 y $60 \mathrm{ml} / \mathrm{min}$ cada $12 \mathrm{~h}$. Los niveles anti-Xa alcanzados estuvieron dentro de las metas predefinidas $(0,6$ a $1,0 \mathrm{UI} / \mathrm{ml})$ con un promedio de $0,65 \mathrm{IU} / \mathrm{ml}$ en los con clearance de creatinina $<30 \mathrm{ml} / \mathrm{min}$ y $0,82 \mathrm{IU} / \mathrm{ml}$ en los con una depuración de creatinina entre 30 y $60 \mathrm{ml} / \mathrm{min}^{46}$. Si bien, una actividad anti-Xa de 0,6-1,0 (peak) con la administración de HBPM cada 12 h ha sido considerada una anticoagulación útil, existe evidencia conflictiva respecto a la correlación entre actividad anti-Xa y riesgo de sangrado ${ }^{47}$.

Al utilizar enoxaparina (ENX) en dosis profilácticas de $40 \mathrm{mg} /$ día por vía subcutánea en pacientes con clearance de creatinina $\leq 30 \mathrm{ml} / \mathrm{min}$, los niveles de actividad anti-Xa están elevados en $40 \%$ respecto a los sujetos con una función renal superior ${ }^{48}$. En un estudio reciente, en que se utilizó dalteparina en una dosis diaria como profilaxis de enfermedad tromboembólica en pacientes con ERC en etapas 3 y 4 , no se encontró una acumulación de la droga superior a 30\% en un seguimiento promedio de 10 días ${ }^{49}$.

Estudios observacionales han comunicado un aumento de los sangrados con HBPM en sujetos con ERC en etapas 3 y 4, algunos de los cuales han sido fatales ${ }^{5,36,50}$ (Tabla 3 ).

El uso de enoxaparina en pacientes con insuficiencia renal severa (clearance de creatinina $<30$ $\mathrm{ml} / \mathrm{min}$ ) aumenta el riesgo de sangrados mayores $(5,4 \%$ vs $2,0 \%)$ y al ajustar las dosis de ENX de acuerdo al clearance de creatinina o a los niveles de actividad anti-Xa, este riesgo se reduce ${ }^{51}$. 
Heparinas de bajo peso molecular en pacientes con enfermedad renal crónica ¿Es seguro su uso? - J. Vega S. et al

Tabla 3. Comunicaciones de hemorragias mayores asociadas al uso de HBPM y ERC

\begin{tabular}{|c|c|c|c|c|c|c|}
\hline Tipo de Sangrado & Droga & Dosis & Indicación & Otras drogas & Evolución & Edad \\
\hline HRP & ENX & $80 \mathrm{mg} \mathrm{c} / 12 \mathrm{~h}$ & TVP & AAS & Muerto & 69 \\
\hline HRP & ENX & $1 \mathrm{mg} / \mathrm{kg} \mathrm{c} / 12 \mathrm{~h}$ & IAM No Q & AAS & Muerto & 83 \\
\hline HRP & ENX & $80 \mathrm{mg} \mathrm{c} / 12 \mathrm{~h}$ & TVP & No & Vivo & 68 \\
\hline HRP & ENX & $60 \mathrm{mg} \mathrm{c} / 12 \mathrm{~h}$ & TVP & No & Vivo & 45 \\
\hline Hemotórax / HRP & ENX & $70 \mathrm{mg} \mathrm{c} / 12 \mathrm{~h}$ & EP & No & Vivo & 58 \\
\hline H. subdural & ENX & $60 \mathrm{mg} \mathrm{c} / 12 \mathrm{~h}$ & SCA / FA & No & Muerto & 82 \\
\hline HRP & ENX & $100 \mathrm{mg} \mathrm{c} / 12 \mathrm{~h}$ & SCA & AAS, AINE'S & Muerto & 80 \\
\hline HRP & NAD & NA & FA & No & Vivo & 72 \\
\hline HRP & ENX & $60 \mathrm{mg} \mathrm{c} / 12 \mathrm{~h}$ & Trombofilia & $\mathrm{ACE}$ & Vivo & 68 \\
\hline HRP & ENX & $80 \mathrm{mg} \mathrm{c} / 12 \mathrm{~h}$ & SCA & AAS, WAR & Vivo & 70 \\
\hline HRP & ENX & $80 \mathrm{mg} \mathrm{c} / 12 \mathrm{~h}$ & SCA & AAS & Vivo & 71 \\
\hline HRP & ENX & $70 \mathrm{mg} \mathrm{c} / 12 \mathrm{~h}$ & IAM No Q & AAS & Vivo & 86 \\
\hline HRP & ENX & $70 \mathrm{mg} \mathrm{c} / 12 \mathrm{~h}$ & IAM No Q & AAS & Vivo & 76 \\
\hline H. del muslo & ENX & $70 \mathrm{mg} \mathrm{c} / 12 \mathrm{~h}$ & EP & No & Vivo & 74 \\
\hline H. de la pierna & NAD & $5.700 \mathrm{IU} \mathrm{c} / 12 \mathrm{~h}$ & E. P. / FA & AAS & Muerto & 81 \\
\hline HRP & DAL & 15.000 IU día & TVP & No & Vivo & 84 \\
\hline HRP & ENX & $45 \mathrm{mg} \mathrm{c} / 12 \mathrm{~h}$ & ATE EEII & AAS, CLO, DIP & Vivo & 61 \\
\hline HRP & ENX & $120 \mathrm{mg} \mathrm{c} / 12 \mathrm{~h}$ & FA & AAS, WAR & Muerto & 74 \\
\hline HRP & ENX & $1 \mathrm{mg} / \mathrm{kg} \mathrm{c} / 12 \mathrm{~h}$ & TVP & No & Vivo & 70 \\
\hline H. pared abdomen & ENX & $60 \mathrm{mg} \times 1 \mathrm{vez}$ & EP & No & Vivo & 43 \\
\hline H. epidural & DAL & $5.000 \mathrm{IU} \times 1$ & Profilaxis & CLO & Vivo & 80 \\
\hline HRP & ENX & $60 \mathrm{mg} \mathrm{c} / 12 \mathrm{~h}$ & SCA & $\mathrm{CLO}$ & Vivo & 70 \\
\hline H. perirrenal & NAD & NA & $H D$ & NA & Vivo & 40 \\
\hline H. perirrenal & NAD & NA & $H D$ & NA & Muerto & 59 \\
\hline H. perirrenal & NAD & NA & $H D$ & NA & Vivo & 73 \\
\hline
\end{tabular}

ERC: enfermedad renal crónica; HBPM: heparinas de bajo peso molecular; HRP: hematoma retroperitoneal; H: hematoma; ENX: enoxaparina; NAD: nadroparina; DAL: dalteparina; HD: hemodiálisis; NA: dato no disponible; TVP: trombosis venosa profunda; IAM: infarto agudo al miocardio; EP: embolia pulmonar; SCA: síndrome coronario agudo; FA: fibrilación auricular; ATE EEll: ateroesclerosis de extremidades inferiores; AAS: aspirina; ACE: acenocumarol; WAR: warfarina; AINE's: antiinflamatorios no esteroidales; CLO: clopidogrel; DIP: dipiridamol.

En la insuficiencia renal la vida media de todas las HBPM está prolongada ${ }^{3}$, pero no existe un valor de corte uniforme que sea predictor del riesgo de sangrado, ya que cada molécula es distinta ${ }^{52}$. Un clearance de creatinina $<30 \mathrm{ml} / \mathrm{min}$ se ha usado frecuentemente para identificar a pacientes con riesgo aumentado de acumulación y sangrado, sin embargo, no hay evidencias que soporten la validez de este umbral ${ }^{52}$. En pacientes con ERC leve (clearance de creatinina $60-70 \mathrm{ml} / \mathrm{min}$ ) que han usado enoxaparina por meses se han reportado también sangrados mayores ${ }^{53}$.

Un estudio retrospectivo, que incluyó a todos los pacientes hospitalizados durante un año con clearance de creatinina $<60 \mathrm{ml} / \mathrm{min}$ y que recibieron dosis terapéuticas de HNF o enoxaparina, reportó que la frecuencia de sangrados aumentó a medida que el clearance de creatinina descendió, 
independientemente de la heparina usada. Sin embargo, en los pacientes con clearance de creatinina $\leq 20 \mathrm{ml} / \mathrm{min}$, aquellos que recibieron enoxaparina presentaron una frecuencia de sangrados 2,5 veces mayor ${ }^{54}$. Con el uso de enoxaparina se han reportado complicaciones hemorrágicas hasta en 22\% de los sujetos con función renal normal y en $51 \%$ de los con creatinina $\geq 2,0 \mathrm{mg} / \mathrm{dl}$, requiriendo del aporte de productos sanguíneos 2\% y $30 \%$, respectivamente ${ }^{50}$.

Además de la insuficiencia renal, la edad, el género femenino, el uso de anticoagulantes orales, trombolíticos y antiplaquetarios, favorecen el sangrado asociado a HBPM. El tiempo de sangría es 2 a 3 veces más largo cuando se usan simultáneamente enoxaparina y aspirina ${ }^{55}$. Ningún antiplaquetario es seguro cuando se utiliza conjuntamente con HBPM en pacientes con ERC avanzada $^{55}$.

Por lo tanto, las estrategias para una anticoagulación óptima para los sujetos con ERC 3 y 4 son variadas y no existe un consenso ${ }^{3}$. Hay autores que recomiendan utilizar sólo HNF en estos pacientes, dada la mayor incidencia de fenómenos hemorrágicos con el uso de HBPM y el dudoso beneficio de estas drogas en sujetos con ERC ${ }^{3}$. La HNF tiene una vida más corta, puede ser fácilmente monitorizada con TTPA y puede neutralizarse completamente con sulfato de protamina ${ }^{3}$.

El Colegio Americano de Médicos de Tórax y el Colegio de Patólogos Americanos recomendaron utilizar HNF en pacientes con clearance de creatinina $\leq 30 \mathrm{ml} / \mathrm{min}$ o monitorizar la actividad anti-Xa si se usan las HBPM en estos pacientes ${ }^{47}$. También se ha aconsejado, dado que las diferentes HBPM tienen un perfil farmacocinético propio, que cada hospital utilice un solo tipo de HBPM en pacientes con ERC, independientemente de la indicación clínica, reduciendo la dosis inicial de acuerdo a la velocidad de filtración glomerular y modificando las siguientes dosis de acuerdo a los niveles de actividad anti-Xa ${ }^{3}$.

El año 2003 cambió el prospecto inserto en los envases de enoxaparina, recomendando para los pacientes con clearance de creatinina $<30 \mathrm{ml} / \mathrm{min}$, reducir las dosis a $30 \mathrm{mg} /$ día en profilaxis y a $1 \mathrm{mg} /$ $\mathrm{kg} /$ día en el tratamiento del tromboembolismo venoso, angina inestable e infarto miocárdico no Q (50\% de la dosis utilizada en sujetos con función renal normal $)^{8}$. Otros autores han propuesto, respecto a la enoxaparina, utilizar una dosis inicial de
0,5 a $0,75 \mathrm{mg} / \mathrm{kg}$ en vez de $1,0 \mathrm{mg} / \mathrm{kg}$ y modificar las dosis siguientes basándose en el monitoreo de la actividad anti-Xa, para evitar los sangrados ${ }^{39,43}$.

Se ha sugerido también reducir la dosis inicial de enoxaparina $16 \%$ en ERC etapa 3 y $44 \%$ en etapa 4, monitorizando la actividad anti-Xa para evitar su acumulación ${ }^{5,33}$. Si bien las dosis ajustadas de HBPM podrían reducir el riesgo de sangrado en pacientes con ERC, ello necesita ser clínicamente evaluado y validado con trabajos prospectivos ${ }^{51}$.

En suma, hasta esta fecha, no se han publicado guías clínicas lo suficientemente claras respecto a la reducción de las dosis o a la forma de monitoreo del uso de las HBPM en pacientes con ERC.

\section{HBPM en pacientes en hemodiálisis}

La HNF continúa siendo la droga más frecuentemente usada para contrarrestar la tendencia de la sangre a coagular en el circuito extracorpóreo durante la hemodiálisis (HD) ${ }^{31}$. Existen varios esquemas para su uso: bolos repetidos, infusión continua y combinaciones de ambos ${ }^{3}$. Debido a las variaciones individuales en la farmacocinética de la HNF, se puede monitorizar el efecto anticoagulante con la medición del TTPA o el tiempo de coagulación activado (ACT) después de la aplicación de un bolo endovenoso inicial ${ }^{3}$, si bien no es una práctica común el monitorizar rutinariamente el efecto anticoagulante en los sujetos en hemodiálisis crónica.

En los pacientes dializados también existen indicaciones de heparina no relacionadas con el procedimiento dialítico, como la enfermedad tromboembólica, enfermedad coronaria y fibrilación auricular ${ }^{31}$.

En Alemania han sido aprobadas 4 HBPM diferentes (enoxaparina, nadroparina, dalteparina y tinzaparina) para su uso en HD o hemofiltración (HF), aunque no existen estudios comparativos disponibles entre las diversas HBPM en $\mathrm{HD}^{3}$. En Estados Unidos de Norteamérica, ninguna se ha aprobado para esta indicación ${ }^{31}$.

Las HBPM no son eliminadas en forma relevante a través de la hemodiálisis o hemofiltración ${ }^{56}$. La presencia de complejos HBPM-antitrombinaFactor Xa y la carga eléctrica negativa pueden explicar la baja permeabilidad de las membranas de diálisis a ellas ${ }^{3}$. La baja eliminación de las HBPM después de una inyección en bolo lleva a 
una anticoagulación sostenida, por lo que todos los fabricantes han recomendado utilizar una sola dosis al inicio de la sesión de $\mathrm{HD}^{3}$.

Una concentración de actividad anti-Xa suficiente $(>0,4 \mathrm{IU} / \mathrm{ml})$ ha sido encontrada hasta $10 \mathrm{~h}$ después de una dosis única de enoxaparina ${ }^{57}$. Con el uso de tinzaparina y reviparina, la disminución de la actividad anti-Xa es más rápida ${ }^{56-58}$.

No existe evidencia de que las HBPM ofrezcan alguna ventaja sobre la HNF para la anticoagulación durante la hemodiálisis ${ }^{3}$. Un metaanálisis de trabajos que utilizaron HBPM en hemodiálisis mostró que el tiempo en lograr la detención del sangrado en el sitio del acceso vascular y la capacidad de evitar la coagulación del circuito fue similar respecto a la HNF, sin embargo, los estudios fueron muy heterogéneos ${ }^{38}$. En un ensayo hecho en Chile con enoxaparina se encontraron los mismos resul$\operatorname{tados}^{59}$. Ello no significa que las HBPM puedan ser usadas en la terapia de las trombosis en pacientes con falla renal, debido a que esta indicación no fue evaluada en el metaanálisis.

Las dosis endovenosas de HBPM durante la hemodiálisis para la prevención de la coagulación del circuito resultan en una anticoagulación de menor duración que las logradas con dosis subcutáneas administradas para anticoagulación terapéutica. Las menores tasas de sangrados mayores observadas en estudios controlados probablemente reflejan las dosis relativamente más bajas empleadas y su administración intermitente ${ }^{38}$.

Al comparar HNF 50 IU/kg como dosis inicial en bolo seguida de 1.000 UI/hora versus enoxaparina $1 \mathrm{mg} / \mathrm{kg}$ en un solo bolo inicial previo a la diálisis, la enoxaparina produjo una menor formación de coágulos en el circuito y una mayor incidencia de sangrados menores $(2,8 \%$ vs $7,7 \%)$, principalmente originados en el acceso vascular. Esto se redujo al utilizar una dosis de $0,69 \mathrm{mg} /$ $\mathrm{kg}^{60}$. Resultados similares, pero sin aumento en los sangrados han sido reportados por otros autores ${ }^{31}$.

Con el empleo de tinzaparina no sólo encontró una reducción en la coagulación del circuito sino también una menor frecuencia de sangrado en el acceso vascular, al compararla con HNF ${ }^{61}$.

Las dosis únicas empleadas pre-diálisis han sido entre 2.500 y 4.250 UI de tinzaparina ${ }^{62,63}, 70$ $\mathrm{UI} / \mathrm{kg}$ de dalteparina ${ }^{50}, 3.075$ a $12.500 \mathrm{UI}$ de nadroparina $^{64-66}$ y 0,5 a 1,0 mg/kg de enoxaparina ${ }^{56,67,68}$.

Es posible que la enoxaparina pueda tener otros metabolitos que potencien su efecto anticoagulan- te y que favorezca el sangrado en sujetos con falla renal ${ }^{68}$.También, cuando se usa repetidamente en hemodiálisis tiene el riesgo de acumularse ${ }^{38}$.

Las dosis precisas de HBPM a emplear en la hemodiálisis no se conocen con exactitud, ya que la mayoría de los ensayos comunicados han usado variedades distintas de HBPM y dosis diferentes $^{31,56}$. Es por ello, que los pacientes con falla renal continúan hemodializándose mayoritariamente con HNF, a pesar del empleo preferente de las HBPM en otras indicaciones de anticoagulación ${ }^{38}$. Se espera que próximamente se desarrollen estudios controlados más rigurosos que permitan recomendar o desaconsejar con mayor fundamento el uso de HBPM para la anticoagulación durante la hemodiálisis ${ }^{38}$. También debe considerarse que el sulfato de protamina no inactiva completamente a las HBPM, por lo que la reversión de su efecto es impredecible ${ }^{69}$.

En suma, la prescripción de HBPM como antitrombótico es cada vez más frecuente debido a su facilidad de uso y comprobada eficacia. Lamentablemente, la mayoría de los estudios que han evaluado la utilidad y seguridad de estas drogas han excluido a los pacientes con ERC, en quienes el catabolismo de estas drogas está reducido con el consecuente aumento de su actividad anticoagulante, principalmente enoxaparina y nadroparina. En forma creciente se han comunicado en ellos eventos hemorrágicos mayores o fatales con su uso (Tabla 3). Hasta el momento, no se ha establecido un nivel de corte en la función renal, donde deba modificarse o evitarse el uso de HBPM, es por ello, que nuestra opinión es desaconsejar el uso de estas drogas en sujetos con ERC en etapas 3 a 5, hasta que aparezcan evidencias más contundentes en la literatura médica de su beneficio y seguridad en este tipo de pacientes.

\section{Referencias}

1. Gould MK, Dembitzer AD, Doyle RL, Hastie TJ, Garber AM. Low-molecular-weight heparins compared with unfractionated heparin for treatment of acute vein thrombosis. A meta-analysis of randomized, controlled trials. Ann Intern Med 1999; 130: 800-9.

2. Antman EM, Morrow DA, Mccabe CH, Murphy SA, Ruda M, Sadowski Z, et al. Enoxaparin versus unfractionated heparin with fibrinolysis for ST-elevation myocardial infarction. N Engl J Med 2006; 354: 1477-88.

3. Hetzel GR, Sucker C. The heparins: all a nephrologist 
should know. Nephrol Dial Transplant 2005; 20: 203642.

4. Antman EM, Cohen M, Radley E, Mccabe C, Rush J, Premmereur J, et al. Assessment of the treatment effect of enoxaparin for unstable angina/non-Q-wave myocardial infarction. TIMI 11B-ESSENCE meta-analysis. Circulation 1999; 100: 1602-8.

5. Farooq V, Hegarty J, Chandrasekar T, Lamerton EH, Mitra S, Houghton, et al. Serious adverse incidents with the usage of low molecular weight heparins in patients with chronic kidney diseases. Am J Kidney Dis 43: 5317.

6. Levey AS, Coresh J, Balk E, Kausz AT, Levin A, Steffes MV, et al. National Kidney Foundation practice guidelines for chronic kidney disease: evaluation, classification, and stratification. Ann Intern Med 2003; 139: 137-47.

7. Antonelli D, Fares L. $2^{\text {nd }}$, Anene C. Enoxaparin associated with hugh abdominal wall hematomas: a report of two cases. Am Surg 2000; 66: 797-800.

8. Groeneveld JH, Van Buren M, Van Overhagen H. Spontaneous renal bleeding in haemodialysis patients. Ned Tijdschr Geeneskd 2008; 152: 1789-93.

9. Kravitz MS, Mishaal RA, Shoenfeld Y. Renal failure and low molecular weight heparins. A dangerous liaison? The case of retroperitoneal hematoma. Isr Med Assoc J 2005; 7: 600-1.

10. Aveline C, Bonnet F. Delayed retroperitoneal haematoma after failed lumbar plexus block. Br J Anaesth 2004; 93: 589-91.

11. Montoya JP, Pokala N. Retroperitoneal hematoma and enoxaparin. Ann Intern Med 1999; 131: 796-7.

12. Chan-Tack KM. Fatal spontaneous retroperitoneal hematoma secondary to enoxaparin. South Med J 2003; 96: 58-60.

13. Dabney A, Bastani B. Enoxaparin-associated severe retroperitoneal bleeding and abdominal compartment syndrome: a report of two cases. Intensive Care Med 2001; 27: 1954-7.

14. Mrug M, Mishra PV, Lusane HC, Cunningham JM, Alpert MA. Hemotorax and retroperitoneal hematoma after anticoagulation with enoxaparin. South Med J 2002; 95: 936-8.

15. Power GE, Rogers P. Retroperitoneal haematoma associated with low molecular weight heparin. Anaesth Intensive Care 2002; 30: 665-7.

16. Gómez de La Torre R, Milla Crespo A, Cadenas F, Fernández Bustamante J, Vásquez Castañón M. Spontaneous retroperitoneal hematoma induced by enoxaparin to therapeutic dose. An Med Interna (Madrid) 2003; 20: 386-7.

17. Melde SL. Enoxaparin-induced retroperitoneal hema- toma. Ann Pharmacother 2003; 37: 822-4.

18. Uthamalingam S, Vadnekar A, Brensilver JM. Enoxaparin-associated spontaneous retroperitoneal hematoma in elderly patients with impaired creatinine clearence: A report of two cases. J Am Geriatr Soc 2004; 52: 477-9.

19. González C, Penado S, Llata L, Valero C, Riancho J. The clinical spectrum of retroperitoneal hematoma in anticoagulated patients. Medicine 2003; 82: 257-62.

20. Cherfan A, Arabi Y, Al Askar A, Al Shimemeri A. Recombinant activated factor VII treatment of retroperitoneal hematoma in a patient with renal failure receiving enoxaparin and clopidogrel. Pharmacotherapy 2007; 27: 755-9.

21. Olson S, Rosatto R, Guazzo E. Acute subdural haematomas and enoxaparin. J Clin Neurosci 2002; 9: 256-9.

22. Balbay O, Tuzuner T, Arbak P, Orhan Z, Erbas M, Aydogan I. Spontaneous leg haematoma in a patient anticoagulated with nadroparin for suspected pulmonary thromboembolism. Swiss Med Wkly 2004; 134: 110-1.

23. Egger S, Sawatzki M, Drewe J, Krahenbuhl S. Lifethreatening hemorrhage after dalteparin therapy in a patient with impaired renal function. Pharmacotherapy 2005; 25: 881-5.

24. Weinsheimer R, Libby E, Howdieshell T. Life-threatening abdominal wall hematoma in a chronic renal failure patient after a single dose of enoxaparin. Am Surg 2005; 71: 132-4.

25. Tam NLK, Pac-Soo C, Pretorius PM. Epidural haematoma after a combined spinal-epidural anaesthesic in a patient treated with clopidogrel and dalteparin. $\mathrm{Br} \mathrm{J}$ Anaesth 2006; 96: 262-5.

26. Antón E, Marti J. Enoxaparin-associated spontaneous thigh haematoma. Age Ageing 2004; 33: 641-2.

27. Hirsh J, Warkentin TE, Raschke P, Granger C, Ohman EM, Dalen JE. Heparin and low-molecular- weight heparin: mechanisms of action, pharmacokinetics, dosing, monitoring, efficacy, and safety. Chest 2001; 119 (Suppl): 64S-94S.

28. Dawes J, Paper DS. Catabolism of low dose heparin in man. Thromb Res 1979; 14: 845-60.

29. Weitz JI. Low-molecular-weight heparins. N Engl J Med 1997; 337: 688-98.

30. La Pointe KJ Cappuzzo KA, Hamilton CW. Low molecular weight heparins for deep vein trombosis. Disponible en: www.uspharmacist.com/oldformat. asp?url=newlook/files/feat/lmwh.htm (consultado el 19 de enero de 2009).

31. Sonawane S, Kasbekar N, Berns J. The safety of heparins in end-stage renal disease. Semin Dial 2006; 19: 305-10.

32. Frydman A. Low-molecular-weight heparins: an overview of their pharmacodynamics, pharmacokinetics and 
metabolism in humans. Haemostasis 1996; 26 (Suppl 2): 24-38.

33. Fareed J, Jeske W, Hoppensteadt D, Clarizio R, Walengo JM. Low-molecular-weight heparins: pharmacological profile and product differentiation. Am J Cardiol 1998; 82 (5B): 3L-10L.

34. Kruzel-Dávila E, Frajewicki V, Kushnir D, Eyal A, Kohan R. Retroperitoneal hematoma in a hemodialysis patient receiving low molecular weight heparin. Isr Med Assoc J 2005; 7: 611-2.

35. Warkentin TE, Levine MN, Hirsh J, Horsewood P, Roberts RS, Gent M, et al. Heparin-induced thrombocytopenia in patients treated with low-molecular-weight heparin or unfractionated heparin. N Engl J Med 1995; 332: 1330-5.

36. Samama MM, Gerotziafas GT. Comparative pharmacokinetics of LMWHs. Semin Thromb Hemost 2000; 26 (supp 1): S31-8.

37. Hulot JS, Vantelon C, Urien S, Bouzamondo A, Mahë I, Ankri A, et al. Effect of renal function on the pharmacokinetics of enoxaparin and consecuences on dose adjustement. Ther Drug Monit 2004; 26: 305-10.

38. Lim W, Cook DJ, Crowther MA. Safety and efficacy of low molecular weight heparins for hemodialysis patients with end-stage renal failure: Meta-analysis of randomized trials. J Am Soc Nephrol 2004; 15: 3192-206.

39. Chow SL, Zammit K, West K, Dannenhoffer M, LópezCandales A. Correlation of antifactor Xa concentrations with renal function in patients on enoxaparin. J Clin Pharmacol 2003; 43: 586-90.

40. Goudable C, Saivin S, Houin G, Sie P, Boneu B, Tonthat $\mathrm{H}$, et al. Pharmacokinetics of a low molecular weight heparin (Fraxiparine) in various stages of chronic renal failure. Nephron 1991; 59: 543-5.

41. Mismetti P, Laporte-Simitsidis S, Navarro C, Sié P, D'azemar P, Necciari J, et al. Aging and venous thromboembolism influence the pharmacodynamics of the anti-factor $\mathrm{Xa}$ and anti-thrombin activities of a low molecular weight heparin (nadroparin). Thromb Haemostasis 1998; 79: 1162-5.

42. Pautas E, Gouin I, Bellot O, Andreux JP, Siguret V. Safety profile of tinzaparin administered once daily at a standard curative dose in two hundred very elderly patients. Drug Saf 2002; 25: 725-33.

43. Becker RC, Spencer FA, Gibson M, Rush JE, Sanderink G, Murphy SA, et al. Influence of patient characteristics and renal function on factor Xa inhibition pharmacokinetics and pharmacodynamics after enoxaparin administration in non-ST segment elevation in acute coronary syndromes. Am Heart J 2002; 143: 753-9.

44. Sanderink GJ, Guimart C, Jariwala N. Enoxaparin phar- macokinetics and pharmacodynamics in renal impairement. J Coll Cardiol 2001; 37: 229A.

45. Peng YG, Eikelboom JW, Tenni P, Mcquillan A, Thom J. Renal function peak anti-Xa levels and enoxaparin dosing. J Pharma Practice Res 2004; 34: 14-7.

46. Kruse MW, Lee JJ. Retrospective evaluation of a pharmacokinetic program for adjusting enoxaparin in renal impairement. Am Heart J 2004; 148: 582-9.

47. Laposata M, Green D, Van Cott EM, Barrowcliffe TW, Goodnight SH, Sosolik RC. College of American Pathologists Conference XXXI on laboratory monitoring of anticoagulant therapy: the clinical use and laboratory monitoring of low-molecular-weight heparin, danaparoid, hirudin and related compounds, and argatroban. Arch Pathol Lab Med 1998; 122: 799-807.

48. Sanderink GJ, Guimart CG, Ozoux ML, Jariwala NU, Shukla UA, Boutouyrie BX. Pharmacokinetics and pharmacodynamics of the prophylactic dose of enoxaparin once daily over 4 days in patients with renal impairment. Thromb Res 2002; 105: 225-31.

49. Schmid P, Brodmann D, Fischer AG, Wuillemin WA. Study of bioaccumulation of dalteparin at a prophylactic dose in patients with various degrees of impaired renal function. J Thromb Haemost 2009;7: 552-8.

50. Gerlach AT, Pickworth KK, Seth SK. Enoxaparin and bleeding complications: A review in patients with and without renal insufficiency. Pharmacotherapy 2000; 20: 771-5.

51. Lim W, Dentali F, Eikelboom JW, Crowther MA. MetaAnalysis: low-molecular-weight heparin and bleeding in patients with severe renal insufficiency. Ann Intern Med 2006;144: 673-84.

52. Nagge J, Crowther M, Hirsh J. Is impaired renal function a contraindication to the use of low-molecular-weight heparin? Arch Intern Med 2002; 162: 2605-9.

53. Busby LT, Weyman A, Rodgers GM. Excesive anticoagulation in patients with mild renal insufficiency receiving long-term therapeutic enoxaparin. Am J Hematol 2001; 67: 54-6.

54. Thorevska N, Amoateng-Adjepong Y, Sabahi R, Schiopescu I, Salloun A, Muralidharan V, et al. Anticoagulation in hospitalized patients with renal insufficiency. A comparison of bleeding rates with unfractionated heparin vs enoxaparin. Chest 2004; 125: 856-63.

55. Malik A, Capling R, Bastani B. Enoxaparin-associated retroperitoneal bleeding in two patients with renal insufficiency. Pharmacotherapy 2005; 25:769-72.

56. Klingel R, Schwarting A, Lotz J, Eckert M, Hohmann V, Hafner G. Safety and efficacy of single bolus anticoagulation with enoxaparin for chronic hemodialysis. Results of an open-label post-certification study. Kidney Blood 
Press Res 2004; 27: 211-7.

57. Guillet B, Simon N, Sampol JJ, Lorec-Panet AM, Portugal H, Berland Y, et al. Pharmacokinetics of low molecular weight heparin enoxaparin during $48 \mathrm{~h}$ after bolus administration as an anticoagulant in haemodialysis. Nephrol Dial Transplant 2003; 18: 2348-53.

58. Hainer JW, Sherrard DJ, Swan SK, Barret JS, Assaid CA, Fossler MJ, et al. Intravenous and subcutaneous weightbased dosing of the low molecular weight heparin tinzaparin (Innohep) in end-stage renal disease patients undergoing chronic hemodialysis. Am J Kidney Dis 2002; 40: 531-8.

59. Vukusich A, Avalos C, Orellana G, Cifuentes C, Rivas J, Calderón F. Anticoagulation in hemodialysis with a single dose of low molecular weight heparin. Rev Med Chile 1995; 123: 735-41.

60. Saltissi D, Morgan C, Westhuyzen J, Healy H. Comparison of low-molecular-weight heparin (enoxaparin sodium) and standard unfractionated heparin for haemodialysis anticoagulation.Nephrol Dial Transplant 1999; 14: 2698-703.

61. Lord H, Jean N, Dumont M, Kassis J, Leblanc M. Comparison between tinzaparin and standard heparin for chronic hemodialysis in a Canadian center. Am J Nephrol 2002; 22: 58-66.

62. Bramham K, Varrier M, Asgari E, Makanjuola D. Comparison of tinzaparin and unfractionated heparin as anticoagulation on haemodialysis: equal safety, efficacy and economical parity. Nephron Clin Pract 2008; 110: c107-13.

63. Egfiord M, Rosenlund L, Hedegaard B, Buchardt HL, Stengel C, Gardar P, et al. Dose titration study of tinzaparin, a low molecular weight heparin, in patients on chronic hemodialysis. Artif Organs 1998; 22: 633-7.

64. Kerr PJ, Mattingly S, Lo A, Atkins RC. The adequacy of fragmin as a single bolus dose with reused dialysers. Artif Organs 1994; 18: 416-9.

65. Lai Kn, Ho K, Li M, Szeto CC. Use of single dose lowmolecular-weight heparin in long hemodialisis. Int J Artif Organs 1998; 21: 196-200.

66. Camici M, Giordani R, Morelli E, Meriggioli M, Balestri $\mathrm{Pl}$, Barsotti G, et al. Safety and efficacy anticoagulation in extracorporeal hemodialysis by simultaneous administration of low-close prostacyclin and low molecular weight heparin. Minerva Med 1998; 89: 405-9.

67. Aggarwal A, Whitaker DA, Rimmer JM, Solomon RJ, Gennari FJ, Sobel BE, et al. Attenuation of platelet reactivity by enoxaparin compared with unfractionated heparin in patients undergoing haemodialysis. Nephrol Dial Transplant 2004; 19: 1559-63.

68. Brophy DF, Wazny LD, Gehr TWB, Comstock TJ, Venitz J. The pharmacokinetics of subcutaneous enoxaparin in end-stage renal disease. Pharmacotherapy 2001; 21: 169-74.

69. Crowther MA, Berry LR, Monagle PT, Chan AK. Mechanism responsible for the failure of protamine to inactivate low-molecular-weight heparin. Br J Haematol 2002; 116: 178-86. 